\section{Neue Generation von Säuglingsnahrungen}

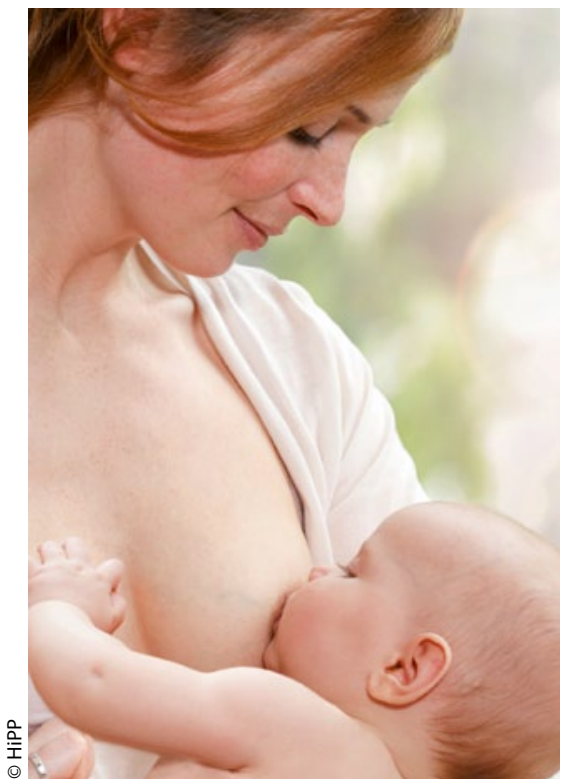

— Orientiert am Vorbild Muttermilch, bietet HiPP als erster Säuglingsnahrungs-Hersteller Anfangsnahrungen mit einer Kombination aus präbiotischen Ballaststoffen und probiotischen Milchsäurekulturen, die aus Muttermilch isoliert wurden: HiPP Combiotik ${ }^{\oplus}$. Dabei ist es erstmals gelungen, die Kombination aus dem Probiotikum Lactobacillus fermentum hereditum ${ }^{\circledast}$, einer aus der Muttermilch isolierten Kultur, und dem Präbiotikum Galacto-Oligosaccharide (GOS) in einer Säuglingsnahrung zu vereinen. Diese neuartige Kombination erwies sich in klinischen Studien als sicher und effektiv und kommt damit den Forderungen von Fachgesellschaften wie der European Society for Pediatric Gastroenterology and Nutrition (ESPGHAN) nach. In

Die Inhalte der Anfangsnahrungen orientieren sich am Vorbild der Muttermilch. einer Studie mit 188 Säuglingen wurden die Folgenahrungen für Kinder zwischen sechs und zwölf Monaten getestet. Das Ergebnis: Säuglinge, die die Kombination aus L. fermentum hereditum ${ }^{\circledast}$ und GOS erhalten hatten, gediehen altersgemäß und erlitten signifikant weniger Durchfälle und Atemwegserkrankungen als die, die Folgenahrung mit ausschließlich GOS erhalten hatten.

Die Nahrungen gibt es ab sofort als HiPP Bio Combiotik ${ }^{\circledast}$ in der bewährten HiPP BioQualität. Sie sind in den Stufen HiPP PRE und HiPP 1 als Anfangsnahrungen von Geburt an mit langkettigen, mehrfach ungesättigten Fettsäuren (LCP) erhältlich. Für das Beikostalter gibt es die HiPP 2 und HiPP 3 Folgenahrungen ab dem sechsten bzw. zehnten Monat. Bei erhöhtem Allergierisiko empfiehlt sich die neue HiPP HA Combiotik ${ }^{\circledR}$ Anfangsund Folgenahrung.

Nach Informationen von HiPP, Pfaffenhoffen

\title{
Was Eltern über Impfungen denken
}

— Die Bundeszentrale für gesundheitliche Aufklärung (BZgA) hat eine repräsentative Studie veröffentlicht, bei der bundesweit 3.000 Eltern zu Kinderimpfungen befragt wurden. Die Mehrheit der Eltern (64\%) lässt in Deutschland ihre Kinder ohne Vorbehalte impfen. Nur 1\% lehnt Kinderimpfungen grundsätzlich ab. Jedoch hat sich mehr als ein Drittel der Befragten (35\%) bereits einmal gegen einzelne Impfungen für ihr Kind entschieden: Knapp die Hälfte von ihnen hielt die ausgelassene Impfung für unnötig, 40\% hatten Angst vor Nebenwirkungen.

„Die Mehrheit der Eltern steht Kinderimpfungen zwar positiv gegenüber. Doch vor allem bei Masern, Keuchhusten und Hepatitis B ist der Impfschutz von vielen Kindern noch immer lückenhaft", betonte Prof. Dr. Elisabeth Pott, Direktorin der BzGA. „Die bevorstehenden Sommermonate, in denen die Kinder seltener krank sind, bieten eine gute Gelegenheit, fehlende Impfungen nachzuholen."

Obwohl wissenschaftlich widerlegt, befürchtet jeder fünfte Befragte, dass Impfungen die Entstehung von Allergien begünstigen können. $68 \%$ aller Eltern glauben zudem immer noch, dass es gut für die Entwicklung ist, wenn das Kind Krankheiten durchstehen muss und mehr als jeder dritte
Erziehungsberechtigte (38\%) hält Masern für nicht gefährlich. Dies ist vor allem vor dem Hintergrund der aktuell stark steigenden Maserninfektionen in Deutschland besorgniserregend.
Die BZgA stellt neben verschiedenen Printmedien auch umfangreiche Informationen auf ihrem Internetportal www.impfen-info. de bereit.

Nach Informationen der Bundeszentrale für gesundheitliche Aufklärung

\section{Aus für die L@us}

Schätzungsweise ein Drittel aller Kopflausinfektionen entfallen auf die Zeit nach den großen Ferien. Rechtzeitig steht mit www.kopflaus.de ein Internetportal bereit, über das sich Ärzte und Apotheker auf den beratungsintensiven saisonalen Höhepunkt dieser Infektionskrankheit vorbereiten können. Praxisinformationen zu Verordnung und Selbstmedikation mit Hilfsmitteln zum Download sind für Fachkreise in einem DocCheckgeschützten Bereich hinterlegt. Die Inhalte sind eng mit den Empfehlungen des RobertKoch-Instituts abgestimmt. Die Website enrhält unter anderem folgende Themen: Epidemiologie, Morphologie, Entwicklungszyklus, Übertragungswege, medikamentöse und ergänzende Behandlungsmöglichkeiten unter Berücksichtigung der wichtigsten heute zur Verfügung stehenden Präparate und Wirkstoffe, ihre jeweilige Wirkweise, Verordnungsfähigkeit, Wirtschaftlichkeit und Anwendung.

Tipps zur Dokumentation sowie Informationen zur Abrechnung auf Basis des aktuellen EBM 2010 unterstützen Ärzte im Hinblick auf eine wirtschaftliche Verordnungspraxis. Im Service-Center der Website finden die Besucher unter anderem Broschüren und mehrsprachige Merkblätter für die Patientenaufklärung. Bei der Website handelt sich um einen Service der Eduard Gerlach GmbH. Das Unternehmen vertreibt seit über 30 Jahren mit Goldgeist forte eines der am häufigsten verwendeten Kopflausmittel in Deutschland.

Nach Informationen von Eduard Gerlach, Lübbecke 